\title{
Understanding eating disorder susceptibility requires an integrated sociological, biological and genetic approach
}

By Dr Jessica K. Edwards

In 2015, Kristen Culbert, Sarah Racine and Kelly Klump compiled a Research Review on the underlying causes of eating disorders for the Journal of Child Psychology and Psychiatry. Their review identified variables that can be considered risk factors for eating pathologies and the critical areas for future research. Here, the researchers revisit their key findings and discuss how the field needs to progress in future years.

Eating disorders are severe psychiatric illnesses with an estimated lifetime prevalence of $\sim 2.8-6.4 \%$ in US adolescents. Three primary categories of eating disorder have been recognized by the DSM-5: anorexia nervosa, bulimia nervosa and binge eating disorder. In addition, 20$40 \%$ cases of eating pathology fall into "Other Specified or Unspecified Feeding or Eating Disorders" categories. Despite diagnostic distinctions between these categories, all are associated with negative medical, cognitive, emotional and social outcomes. As such, increasing our understanding of the factors that contribute to the development of an eating pathology is necessary for early intervention and prevention

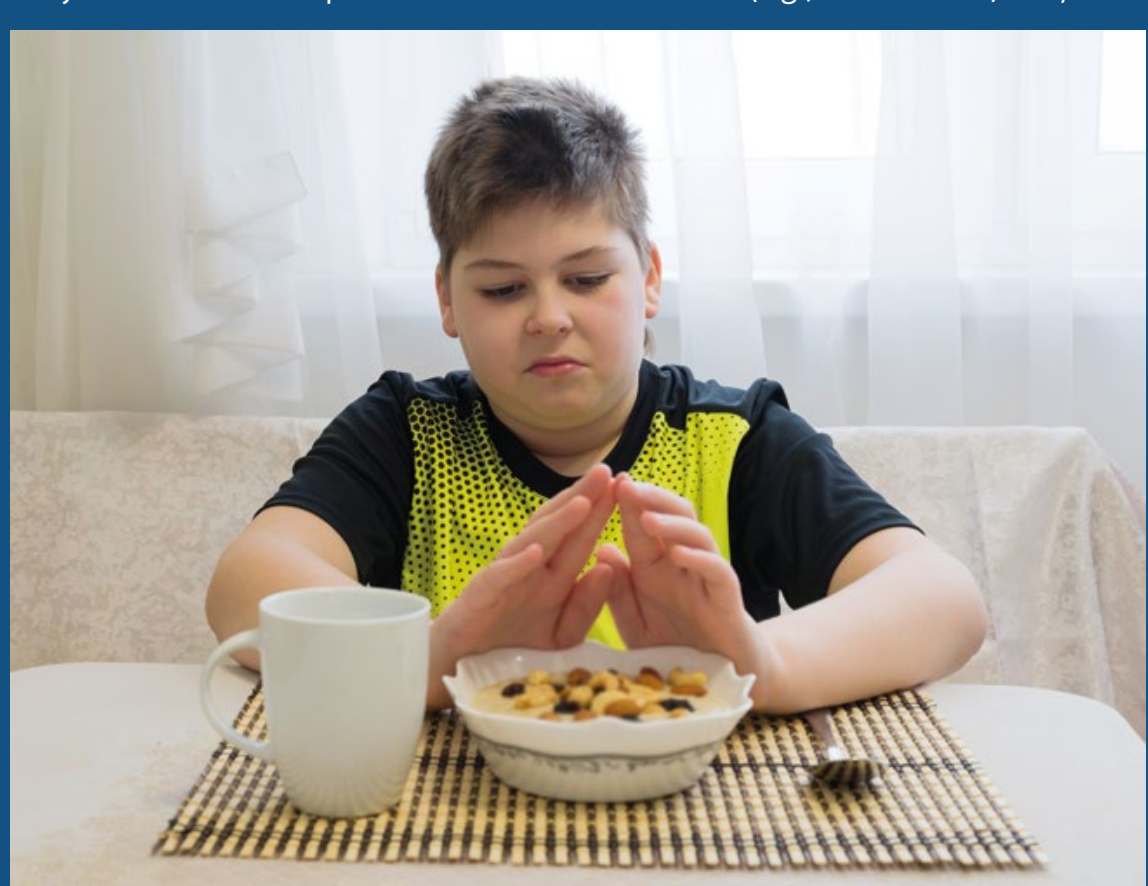

In their review, Culbert and colleagues reinforce that no single factor accounts for the development of an eating disorder. "Risk for eating disorders involves a complex interplay between sociocultural, psychological and biological influences, but most research has examined biological and psychosocial risk factors in isolation", explains Culbert. "Studies that examine factors across all domains are critical to fully determine the causes that contribute to the development of an eating disorder. Consequently, our review aimed to identify key risk factors that had been studied using integrative methods - i.e. approaches that captured both biological and environmental influences (e.g., twin studies) and/ 
